\title{
Article \\ Thermodynamics Modeling for Actinide Monocarbides and Mononitrides from First Principles
}

\author{
Per Söderlind *(D), Emily E. Moore ${ }^{(D)}$ and Christine J. Wu \\ Lawrence Livermore National Laboratory, Livermore, CA 94551-0808, USA; moore255@1lnl.gov (E.E.M.); \\ wu5@llnl.gov (C.J.W.) \\ * Correspondence: soderlind@llnl.gov; Tel.: +1-925-423-4667
}

\begin{abstract}
The high-temperature thermodynamical properties for the actinide monocarbides and mononitrides ThC, ThN, UC, UN, PuC, and PuN are calculated from first-principles electronicstructure theory. The electronic structure is modeled with density-functional theory (DFT) and is fully relativistic, including the spin-orbit interaction. Furthermore, the DFT is extended to account for orbital-orbital interactions, by means of a parameter-free orbital-polarization (OP) technique, that has proven to be essential for the $5 \mathrm{f}$ electrons in plutonium. Strong anharmonicity and the temperature dependence of the lattice vibrations are captured with the self-consistent ab initio lattice dynamics (SCAILD) method. The calculated free energies and heat capacities are compared to published results from quasi-harmonic $(\mathrm{QH})$ theory, and experiments, where available. For the uranium and plutonium compounds, we make use of CALPHAD assessments to help evaluate the theory. Generally, our anharmonic relativistic approach compares well with both CALPHAD and experiments. For the thorium compounds, our theory is in good accord with QH modeling of the free energy at lower temperatures but for the heat capacity the comparison is less favorable.
\end{abstract}

Keywords: actinide monocarbides; mononitrides; free energy; specific heat; thermodynamics; anharmonic

check for

updates

Citation: Söderlind, P.; Moore, E.E.; $\mathrm{Wu}, \mathrm{C} . J$. Thermodynamics Modeling for Actinide Monocarbides and Mononitrides from First Principles. Appl. Sci. 2022, 12, 728. https:// doi.org/10.3390/app12020728

Academic Editor: Leonid Burakovsky

Received: 6 December 2021

Accepted: 10 January 2022

Published: 12 January 2022

Publisher's Note: MDPI stays neutral with regard to jurisdictional claims in published maps and institutional affiliations.

Copyright: (c) 2022 by the authors. Licensee MDPI, Basel, Switzerland. This article is an open access article distributed under the terms and conditions of the Creative Commons Attribution (CC BY) license (https:// creativecommons.org/licenses/by/ $4.0 /)$.

\section{Introduction}

Actinide carbides and nitrides are of practical importance as possible nuclear fuels for Generation-IV (fast neutron fission) reactors [1,2]. Currently, nuclear fuels are often ceramic fuel pellets of actinide dioxides or actinide mixes of dioxides. More advanced fuels may consist of actinide, primarily uranium and plutonium, carbides and nitrides, due to their greater density and thermal conductivity. Furthermore, they release more neutrons than consumed in the reactor which is beneficial for energy production. However, the suitability of the nuclear carbide and nitride fuels is still being evaluated and fundamental research, experimental and modeling, has increased significantly in recent years [3-18]. These radioactive and toxic compounds are not trivial to work with and their material properties at higher temperatures, central for nuclear fuels, are difficult to measure accurately. From a modeling point of view, the electronic structure is complex, and the high temperatures of interest for fuels cause the atoms to behave in a challenging anharmonic fashion.

The physics models presented to date for the actinide monocarbides and mononitrides have been, with few exceptions, either semi-empirical in nature or restricted to a quasi-harmonic $(\mathrm{QH})$ treatment of the lattice dynamics. The $\mathrm{QH}$ approach, however, is known to be less predictive at higher temperatures $[8,15]$. In this report, we address these shortcomings in modeling by combining advanced first-principles electronic structure with a lattice-dynamics method that can treat strong lattice anharmonicity at high temperatures. For comparison and validation of the modeling, we apply the CALPHAD method to compare the assessments of experimental data in addition to directly contrasting the theory with measurements, where available.

Actinide-bearing materials, including the present potential fuels, are complicated to handle, both theoretically and experimentally. Consequently, some basic properties, 
including details of the magnetism, are not yet well categorized for the actinide carbides and nitrides. For fundamental theory, the challenges often focus on the electronic and crystal structure. In regard to the latter, the $5 \mathrm{f}$ electrons play important roles for all actinides up to plutonium. Even for thorium, that has the least amount of $5 \mathrm{f}$ electrons, their presence dictates the crystal structure [19]. The dominance in the chemical bonding of the $5 f$ electrons is reflected in the gradual increase in complexity of the crystal structures with atomic number up to plutonium that has an extraordinary monoclinic phase [20]. The actinide monocarbides and mononitrides, however, solidify in the sodium chloride $(\mathrm{NaCl})$ or rock salt structure, i.e., a cubic phase with the two atoms in the (000) and (1/2 $1 / 21 / 2)$ positions, respectively. This simple phase, also referred to as $\mathrm{B} 1$, makes the theoretical treatment considerably easier than that of more complex structures.

While the crystal structure is basic for these rock salt compounds, the electronic structures are very intricate and require careful consideration. For uranium and plutonium compounds, particularly oxides, the $5 f$ electrons are often treated in a physics model with density-functional theory (DFT) and a Hubbard U parameter designed to address a large intra-atomic Coulomb repulsion in the DFT + U method. A broad study [21] of actinide oxides, carbides, and nitrides, in the rock salt structure, considered both the $\mathrm{DFT}+\mathrm{U}(\mathrm{U} \sim 4 \mathrm{eV})$ and the screened-hybrid-DFT approaches. In the latter, a chosen arbitrary amount of Hartree-Fock electron exchange interaction is explicitly included in the model. The conclusion from this investigation [21] was that for actinide dioxides these models are appropriate but for the metallic carbides and nitrides, the screened-hybrid-DFT is problematic. Namely, the photoemission for $\mathrm{UN}$ is not correctly reproduced, contrary to the conventional DFT model that compares well with experiment. For the present actinide monocarbides and mononitrides, the $+\mathrm{U}$ approach has not been shown to be effective either. For example, DFT $+\mathrm{U}(\mathrm{U} \sim 2 \mathrm{eV})$ generates imaginary phonon frequencies for $U N$, resulting in a dynamical instability of the compound, and the method was consequently not recommended [18].

A far less practical methodology is that of the dynamical mean-field theory (DMFT) that also relies on the Hubbard $U$ and other parameters. For DMFT, the computational cost accelerates dramatically while the calculated total energy becomes ambiguous because the double-counting energy contribution is not clearly defined. Therefore, energy-derived properties, crucial for thermodynamics, are not accessible from the DMFT method [22]. On a more fundamental level, all $+\mathrm{U}$ (and hybrid-DFT) methods suffer from having one or more parameters that are impossible to know for certain and therefore assumptions must be made when deciding their values. These approaches further rely on using fixed parameters, while in reality they must depend on both temperature and pressure. Unfortunately, the calculated properties often depend rather sensitively on these uncertain parameters [23].

As a better alternative, we have employed an approach that includes orbital polarization (OP) in a DFT + OP method [24], involving self-consistent Racah parameters, where OP accounts for magnetic orbital-moment couplings. This orbital-polarization interaction only exists when electrons are moving (orbiting) and is therefore not explicitly addressed in conventional DFT that is free of charge currents. Results from the present DFT + OP scheme, that is thus an extension of conventional DFT, agree well with those obtained from a method where OP is rigorously derived from current-density-functional theory [25].

It has been argued that there is a connection between the $+\mathrm{U}$ methods and orbital polarization, namely, that DFT + OP can be mimicked by DFT + U in the limit of very small $U$ [26]. This may be approximately true for very small $U$, but the two methods are certainly not equivalent. The OP is driven by an orbital-moment interaction that only exists when the spin-orbit interaction and orbital moments are present while DFT $+\mathrm{U}$ does not depend on spin-orbit coupling. Another more fundamental difference is that DFT + OP is a self-consistent approach that adapts to changing conditions while DFT $+\mathrm{U}$ is not. We have shown that OP is essential for plutonium [27] while less important for thorium and uranium [28], but we have nonetheless chosen to include OP in most of our present calculations because of the small additional computational cost (see discussion below). 
Aside from the general difficulty of the $5 \mathrm{f}$ electronic structure, vacancies (of nitrogen or carbon) tend to form in these materials thus further complicating the modeling of a realistic material. These effects can be modeled at low temperatures [29] but for the present hightemperature study, we do not address any defect mechanisms or dependencies on defects, but rather assume perfect, stochiometric crystals.

Our present results compliment those we have published for uranium mononitride [8] and plutonium monocarbide [15] so that we now can present thermodynamical modeling for a complete set of thorium, uranium, and plutonium monocarbides and mononitrides. The uranium and plutonium compounds (and mixes of them) are considered potential advanced nuclear fuels [1,2]. The thorium compounds we include because they are of interest for very high-temperature reactors [1] but also because they provide a broader fundamental-science context to the results for the other compounds.

\section{Computational Methods}

\subsection{Electronic-Structure Methods}

The electronic structures are obtained from density-functional theory where the necessary electron exchange and correlation is the generalized gradient approximation (GGA). Generally, with some exceptions noted, DFT is extended to include an orbital-polarization mechanism that accounts for electron orbital-orbital coupling that is absent in conventional DFT. For the thorium compounds, the strength of this interaction is limited, but for the magnetic compounds and plutonium, it cannot be ignored $[15,27,28]$. Fortunately, the implementation is efficient, and the computational penalty for including OP is not severe enough to dissuade us from applying the interaction for all presently studied compounds.

We employed two codes for the electronic-structure calculations in this paper. First, for the most accurate electronic structure, we rely on an all-electron full-potential linear muffintin orbital (FPLMTO) method [30]. FPLMTO is used to calculate all electronic structures and energies except the atomistic forces that are needed for the lattice-dynamics method. For computing these forces, we utilized the more expedient pseudopotential plane-wave approach (see below).

The FPLMTO method is characterized by its atomic-like basis functions that for actinides include $6 s, 6 p, 7 s, 7 p, 6 d$, and $5 \mathrm{f}$ orbitals that are allowed to hybridize in one energy window. Some quantities in the method are expanded in series (basis functions, electron densities, and potentials) of spherical harmonics in the muffin-tin sphere position at each atomic site. The radial part of the basis functions inside these spheres are calculated from a wave equation that addresses all relativistic corrections including the spin-orbit coupling for $\mathrm{d}$ and $\mathrm{f}$ states but not for the $\mathrm{p}$ states, as is appropriate and accurate for actinides [28]. Guided by the self-consistent Racah parameters obtained from the FPLMTO method we define one Racah parameter, associated with the $5 \mathrm{f}$ electrons, that is kept fixed $(0.05 \mathrm{eV})$ for use in the pseudopotential method. The theoretical treatment is spin polarized (magnetic) and we found that the ferromagnetic configuration is lower in energy than the most trivial anti-ferromagnetic configuration (a doubling of the unit cell along the $\mathrm{z}$ axis), except for the thorium compounds where the spin moment collapses to zero. For this reason, and the fact that the magnetic moment disorders at very high temperatures, we only consider ferromagnetism in our thermodynamical modeling.

In Table 1, we show our all-electron FPLMTO-calculated atomic volumes for the nonmagnetic (NM), anti-ferromagnetic (AF), and ferromagnetic (FM) configurations together with experimental data [16,31-35]. Overall, the theory reproduces the experiment fairly well, suggesting that GGA is a reasonable choice for these compounds. GGA is not as perfect as it is for the actinide metals [28], but that is not expected for these compounds because at least for carbon, GGA overestimates the atomic volumes significantly [36]. The volumes for all present monocarbides are consequently also overestimated, see Table 1 . The atomic volumes in Table 1 are calculated at zero temperature and not corrected for thermal expansion (measurements are often taken at room temperature). Such a correction is small but will not uniformly improve the agreement between theory and experiment. 
Table 1. The atomic volumes in $\AA^{3}$ /atom from present theory and experimental data [16,31-35].

\begin{tabular}{ccccccc}
\hline Method & ThC & ThN & UC & UN & PuC & PuN \\
\hline Theory-NM & 19.20 & 17.27 & 15.24 & 14.53 & 14.71 & 14.70 \\
Theory-AF & - & - & 15.27 & 14.85 & 16.00 & 15.00 \\
Theory-FM & - & - & 15.33 & 14.85 & 16.19 & 15.18 \\
Experiment & 18.84 & 17.41 & 15.26 & 14.62 & 15.44 & 14.89 \\
\hline
\end{tabular}

For the uranium and plutonium compounds, there are few direct measurements of magnetism but UN and PuC are found to be anti-ferromagnetic [37,38]. The only reported experimental magnetic moment is for $\mathrm{UN}$, and it is $0.75 \mu_{\mathrm{B}}$ [38]. Apparently, the measurements were not of high enough fidelity to reveal the magnetic moment for $\mathrm{PuC}$ [37]. Our anti-ferromagnetic calculations for $\mathrm{PuC}$ and $\mathrm{PuN}$ give atomic volumes closer to experiments, whereas the volumes for $\mathrm{UC}$ and $\mathrm{UN}$ are less sensitive to the magnetic ordering, see Table 1.

For our thermodynamics calculations we assume ferromagnetism, regardless of what the actual (room temperature) configuration may be. This is not an important concern, however, because at the very high temperatures of interest here, magnetic order is entirely lost. Our all-electron FPLMTO-computed (low temperature) magnetic moments are listed in Table 2. Because the $5 \mathrm{f}$ shell is less than half occupied for $\mathrm{U}$ and $\mathrm{Pu}$, the spin and orbital moments align anti-parallel so that the total moment is relatively small for the uranium and plutonium compounds. The orbital moment is significantly enhanced, resulting from the orbital polarization of the $5 \mathrm{f}$ electrons [39], and the OP interaction is therefore essential for good agreement between theory and experiment for the net magnetic moment in UN. The orbital-polarization mechanism is not only important for the magnetic properties, but it is critical also for the thermodynamical properties of $\mathrm{PuC}$ [15].

Table 2. The calculated (low temperature) magnetic moments in units of $\mu_{B}$. The only available experimental value is that of $\mathrm{UN}, 0.75 \mu_{\mathrm{B}}$ [38].

\begin{tabular}{ccccc}
\hline Magnetic Moment & UC & UN & PuC & PuN \\
\hline Spin & -0.04 & -1.31 & -3.81 & 4.10 \\
Orbital & 0.08 & 2.09 & 3.92 & -3.60 \\
Total & 0.04 & 0.78 & 0.11 & 0.50 \\
\hline
\end{tabular}

For the atomic-force calculations, associated with lattice dynamics (see next subsection), we took advantage of an approach computationally cheaper than the more accurate FPLMTO. Namely, the pseudopotential plane-wave Vienna ab initio simulation package (VASP) and the projector-augmented-wave (PAW) method [40-42]. The VASP-PAW computational parameters consist of an energy cut-off of $450 \mathrm{eV}$, energy convergence of $100 \mathrm{eV}$, and a Monkhorst-Pack scheme with a total of $8 \mathrm{k}$ points for the 54-atom supercell we employ for the lattice-dynamics method.

Ferromagnetic order is assumed for the VASP-PAW magnetic calculations, in analogy with the FPLMTO calculations. The electron exchange and correlation functional is that of the GGA and includes, for the plutonium compounds, spin-orbit coupling, and orbital polarization with a fixed 5 f-electron Racah parameter $(0.05 \mathrm{eV})$ as implemented and presented [28]. The value is decided from the Racah parameters that are self-consistently determined within the FPLMTO method.

For comparison with our FPLMTO all-electron results we show VASP-PAW-calculated atomic volumes and spin moments for ThC, ThN, UC, UN, PuC, and PuN in Table 3. Here, the thorium compounds are non-magnetic while the others are treated as ferromagnetic. For these calculations we increased the number of $k$ points to about 400 to better resolve energies and magnetic moments. 
Table 3. Atomic volumes ( $\AA^{3} /$ atom) and spin moments $\left(\mu_{\mathrm{B}}\right)$ from VASP-PAW calculations.

\begin{tabular}{ccccccc}
\hline Property & ThC & ThN & UC & UN & PuC & PuN \\
\hline Atomic volume & 19.25 & 17.52 & 15.55 & 14.91 & 15.87 & 15.10 \\
Spin moment & - & - & 0.685 & 1.246 & 3.571 & 3.869 \\
\hline
\end{tabular}

Overall, the two computational approaches give similar results with some differences that are expected from two relatively different electronic-structure methods. We consider the all-electron results to be most accurate and the FPLMTO method is used to calculate all properties in this paper, except the forces that are utilized in the SCAILD lattice-dynamics scheme.

\subsection{Lattice-Dynamics Method}

For calculations of lattice dynamics, we employed the self-consistent ab initio lattice dynamics (SCAILD) methodology [43]. The idea behind this method is to, in a first step, apply a small displacement to an atom (or atoms, depending on crystal structure) in the supercell of the perfect crystal and calculate the forces on all the atoms. Second, using these forces, in analogy with the finite displacement method, to calculate the phonon density of states in the harmonic approximation. In the third step, one can determine, at a given temperature, the associated atomic displacements from standard lattice-dynamics equations. Next, again calculate the atomic forces on the crystal with thermally excited atoms and lastly, establish the new phonon density of states. The scheme is iterative because it returns to step 3 and self-consistent as it eventually converges so that the phonon density of states and associated properties are no longer changing. For example, the free energy is the most fundamental property that we are focusing on in this report. The convergency of the free energy is thus very important and in $[8,15]$ we showed the lattice contribution to the free energy as a function of SCAILD iterations.

In the present case, for the actinide monocarbides and mononitrides the number of iterations required for convergency of the SCAILD scheme is about 100-200. The entire procedure is carefully explained in mathematical detail [43] and is not repeated here.

We thus applied SCAILD for the six compounds: ThC, ThN, UC, UN, PuC, and PuN. The supercell we chose for this purpose is a 54-atom $(3 \times 3 \times 3)$ cell of the $\mathrm{NaCl}$ structure. Technically, SCAILD assumes fixed lattice constant and temperature so to include volume and temperature dependencies, it is repeated on a volume-temperature grid. We typically executed SCAILD for about 4-6 temperatures and 3-4 volumes per compound. For the calculation of the forces on the atoms we apply density-functional theory as implemented in an efficient plane-wave pseudopotential code, described above. The total number of such DFT atomic-force calculations for this report, including all six compounds, was about 20,000. Albeit a considerable effort, the alternative, quantum molecular dynamics simulations, are far less manageable from a computational standpoint.

\subsection{CALPHAD Method}

We are using the thermodynamical CALPHAD method to establish the heat capacity and Gibbs (free) energy of the uranium monocarbide, mononitride, as well as plutonium monocarbide, and mononitride as functions of temperature. For the thorium compounds we did not perform CALPHAD calculations because for them the experimental data are too sparse and consequently no assessments have been done. In general, the main purpose of CALPHAD is to model the Gibbs energy of various phases relative to binary and ternary systems to best reproduce carefully inspected phase diagrams and thermodynamics. From the Gibbs energy one subsequently determines phase stability and thermodynamical properties of multi-component systems [44-46].

In the present report, the CALPHAD is contrasted with the first-principles modeling. An additional benefit is that the first-principles data improve the robustness of the thermodynamical-modeling capability, particularly for materials that have many unknown variables. CALPHAD has been successful in predicting complex phase transformation in 
plutonium alloys [47,48] and first-principles-informed CALPHAD assessments for actinide systems have become customary in recent years [49-51].

\section{Results}

\subsection{ThC and $T h N$}

For thorium monocarbide and mononitride, as well as their uranium and plutonium counterparts, we adopted an approach combining electronic-structure calculations with lattice dynamics to obtain free energies and specific heats as functions of temperature. We focused on higher temperatures where these materials have not previously been studied to any greater extent. The free energy is not explicitly measured in any experiment, but the CALPHAD technique allows for a determination of this quantity that we can compare with However, due to the scarcity of experimental data, we are not able to make a comparison to CALPHAD data of either ThC or ThN.

Although the free energy can be straightforwardly calculated as a function of volume compression or pressure condition, we are only concerned about the free energy at constant, ambient (zero), pressure in this investigation.

We introduce the most general expression of the Gibbs free energy at zero pressure (or Helmholtz free energy) that we consider:

$$
F(V, T)=F_{\text {lat }}(V, T)+F_{\text {el }}(V, T)+F_{\text {mag }}(V, T) .
$$

Here, $F_{l a t}$ is the free energy from lattice vibrations, $F_{e l}$ the electronic free energy, including entropy, and $F_{m a g}$ is the magnetic contribution due to entropy of magnetic disorder, $S_{\text {mag }}$.

$$
F_{m a g}(V, T)=-k_{B} T S_{m a g}=-k_{B} T \ln (2 \mu+1),
$$

where $k_{B}$ is the Boltzmann constant and $\mu$ the total magnetic moment (spin and orbital contributions) $[52,53]$. The lattice free-energy contribution, $F_{\text {lat }}$, is derived from the VASP-PAWcalculated atomic forces that are coupled with SCAILD, while the other two contributions are obtained from the FPLMTO electronic structure. Density-functional theory predicts no magnetism in the thorium compounds, so the magnetic contribution is only relevant for the uranium and plutonium systems. Table 2 lists the calculated magnetic moments for UC, $\mathrm{UN}, \mathrm{PuC}$, and $\mathrm{PuN}$ that we are using for the free-energy contributions.

In principle, there is also an electron-phonon coupling term in the free energy expression in Equation (1) but there are, to our knowledge, no well-defined or straightforward procedures to calculate it accurately. Nonetheless, electron-phonon coupling is implicitly included in our strategy because all free-energy contributions are evaluated at the equilibrium volume and this volume, in turn, depends strongly on the calculated lattice dynamics.

Lacking CALPHAD free energy for ThC (and ThN, see below), we have chosen to compare our result with published theory that is constrained to quasi-harmonic lattice vibrations [4]. In Figure 1, we show our calculated free energy together with results from quasi-harmonic $(\mathrm{QH})$ modeling up to $900 \mathrm{~K}$. Above $900 \mathrm{~K}$ we have added a cubic-spline extrapolation of the $\mathrm{QH}$ data as an approximate comparison at the highest temperatures.

In our experience, a quasi-harmonic calculation for an actinide compound is often relatively good up to about $1000 \mathrm{~K}$ [8], so we are not surprised to find the same here for ThC. Certainly, at lower temperatures there is no significant difference between our present anharmonic approach and the QH treatment [4]. The Sahafi and Mahdavi model [4] does not go above $900 \mathrm{~K}$ but the extrapolation to higher temperatures suggests that beyond about $1500 \mathrm{~K}$ their $\mathrm{QH}$ model is becoming increasingly inaccurate.

Perhaps a more interesting evaluation of the present theory for the thermodynamics of ThC is to compare the specific heat at constant pressure, $C_{p}$, with available experimental data. However, as already alluded to, there are very limited thermodynamical data on ThC. Actually, we cannot find any measured $C_{p}$ at all for higher temperatures, only up to room temperature by Danan [54]. 


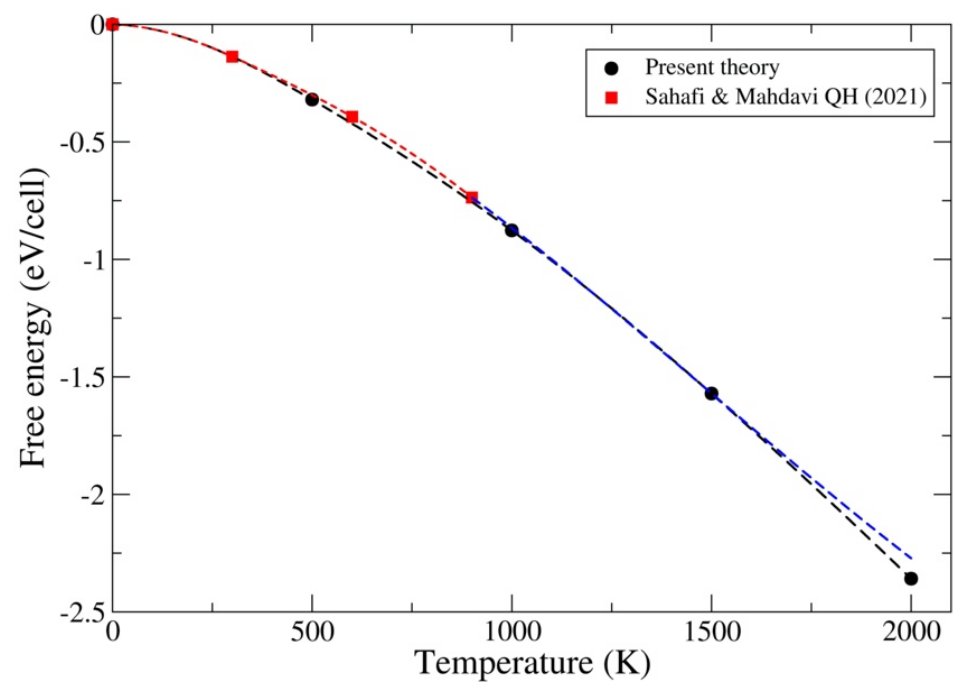

Figure 1. Free energy for ThC from present theory together with results from a first-principles quasiharmonic $(\mathrm{QH})$ model [4] where the blue dashed curve (above $900 \mathrm{~K}$ ) is a cubic-spline extrapolation. Results are solid symbols and dashed lines are guides for the eye only.

Our general approach for calculating $C_{p}$ is to add up the following terms, analogous to the free-energy expression shown in Equation (1):

$$
C_{p}(T)=C_{p}^{\text {lat }}(T)+C_{p}^{e l}(T)+C_{p}^{m a g}(T) .
$$

Here, $C_{p}{ }^{\text {lat }}$ is obtained indirectly from SCAILD because this method is performed at constant volume (and temperature), not at constant pressure. Therefore, a conversion of the heat capacity to constant pressure is required and it can be done in several ways. One can do as we have done for the free energy, namely, calculate the total free energy, i.e., the lattice contribution, $F_{l a t}$, and the electronic contribution, $F_{e l}$, on a volume-temperature grid and establish the equilibrium properties (pressure is zero). Alternatively, one can extract the constant-volume specific heat, $C_{v}^{\text {lat }}$ (from SCAILD) and convert to $C_{p}{ }^{\text {lat }}$ by means of this expression:

$$
C_{p}^{\text {lat }}(T)=C_{v}^{\text {lat }}(T)[1+\gamma \alpha T] .
$$

The temperature-dependent parameters $\gamma$ and $\alpha$, are the Grüneisen and volumeexpansion coefficients, respectively. Both of these quantities are easily obtained from a quasi-harmonic model. The two approaches for determining $C_{p}{ }^{\text {lat }}$ give rather similar results and we are generally using Equation (4) for the conversion.

The electronic contribution to the specific heat, $C_{p}{ }^{e l}$, is derived from the electronic density of states, $D(E)$, at the highest occupied energy state (Fermi level, $\left.E_{F}\right), D\left(E_{F}\right)$ :

$$
C_{p}^{e l}(T)=\frac{\pi^{2}}{3} D\left(E_{F}\right) k_{B}^{2} T .
$$

Note, $D(E)$ depends on temperature because it is subject to a Fermi-Dirac temperature distribution. Thermal volume expansion, that depends on the lattice dynamics, influences $D(E)$ as well. We account for both these effects when determining $C_{p}{ }^{e l}$.

Finally, the magnetic term in Equation (5), $C_{p}{ }^{m a g}$, is acquired from the following thermodynamical equation at constant pressure [55]:

$$
C_{p}^{m a g}(T)=k_{B} T\left[\frac{d S_{m a g}(T)}{d T}\right]_{p}
$$


where the magnetic entropy, $S_{m a g}$, is expressed in Equation (2). The temperature dependence of the total magnetic moment, $\mu$, is derived from the DFT + OP electronic structure for the given temperature and the corresponding equilibrium volume.

In Figure 2, we show our calculated specific heat for ThC with $\left(C_{p}\right.$-lat-el) and without $\left(C_{p}\right.$-lat $)$ the electronic part, together with two quasi-harmonic models $[3,5]$ and experimental data from Danan [54]. Unfortunately, the specific heat is measured at lower temperatures than we have studied, but at least we can compare our results with the two quasi-harmonic approaches [3,5]. It appears that one [3] of them is consistent with our present theory while the other [5] is not. The two sets of $\mathrm{QH}$ results are actually in disagreement in a wide temperature range, and that is indicative of the general uncertainty associated with the quasi-harmonic technique.

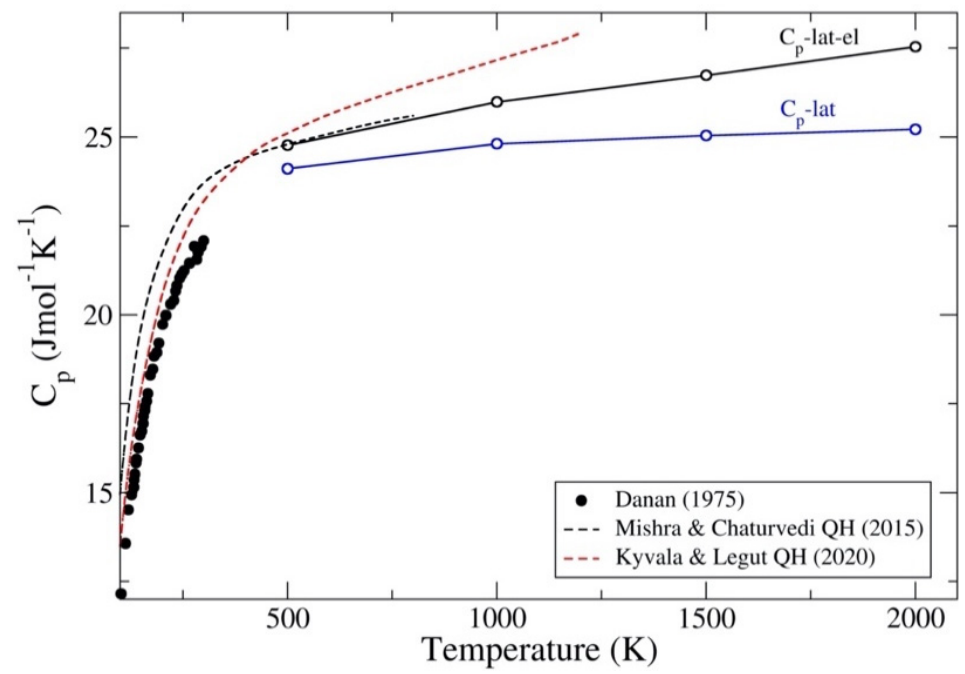

Figure 2. Specific heat at constant pressure, $C_{p}$, for ThC. Levels of theory are open circles, where the connecting line is a guide for the eye only. The dashed lines refer to quasi-harmonic $(\mathrm{QH})$ modeling [3,5]. Solid symbols are from experiment [54].

It is clear from our results in Figure 2 that the electronic contribution to the specific heat is significant and cannot be ignored. This contribution is proportional to the electronic density of states at the Fermi level, $D\left(E_{F}\right)$, as expressed in Equation (5). This quantity directly reflects the electronic structure, and it is very sensitive to the fundamental theory as well as details of the calculation.

Including the physics of the intra-atomic Coulomb repulsion, that has been suggested for actinide compounds, would likely (depending on the choice of Hubbard U parameter) lower $D\left(E_{F}\right)$ rather dramatically. Spin polarization also has a substantial effect of lowering $D\left(E_{F}\right)$ relative to a spin degenerate (non-magnetic) treatment of the electronic structure. In a similar way as spin polarization, spin-orbit coupling and orbital polarization play roles for the electronic specific heat as well.

The all-electron FPLMTO calculations we include orbital polarization for all six compounds, while OP is only included in the VASP-PAW force calculations (for lattice dynamics) for PuC and PuN and not for the thorium or uranium compounds (for them the OP effect is much smaller). The OP interaction is analogous to the spin-orbit coupling and helps to lower $D\left(E_{F}\right)$ because of the increased energy separation between electronic states with different magnetic quantum number $\left(m_{l}\right)$.

Next, we discuss our results for thorium mononitride, ThN. As in the case of ThC, we have no CALPHAD results to compare with but for the free energy we instead contrast our results with a quasi-harmonic model [11]. Again, the $\mathrm{QH}$ theory appears to be close to our anharmonic model up to about $1000 \mathrm{~K}$ but increasingly less accurate beyond that, assuming a simple extrapolation. 
Our predicted free energies (Figures 1 and 3) at higher temperatures can be helpful for future CALPHAD assessments or for constraining semi-empirical approaches for $\mathrm{ThC}$ and $\mathrm{ThN}$.

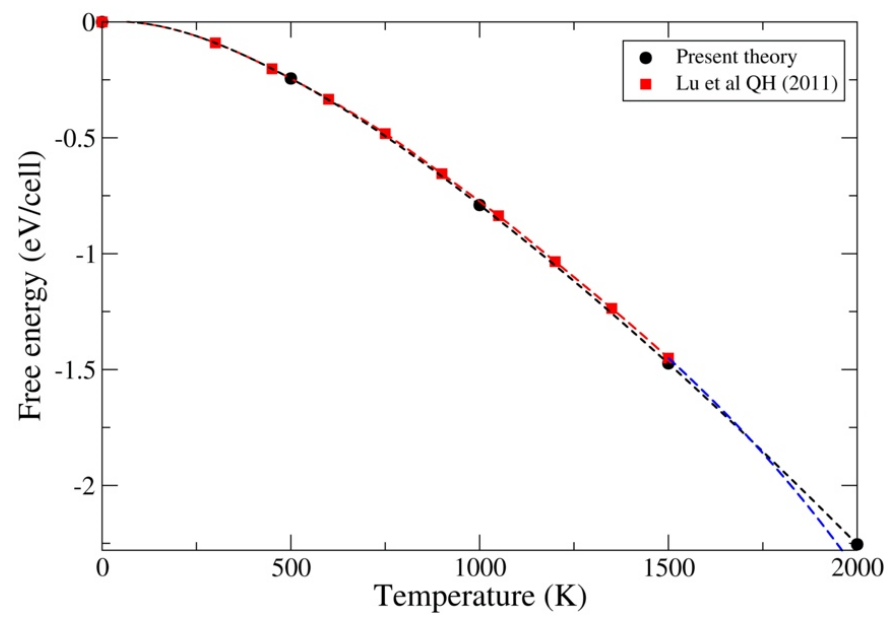

Figure 3. Free energy for ThN from present theory together with results from a first-principles quasiharmonic (QH) model [11] where the blue dashed curve (above $1500 \mathrm{~K}$ ) is a cubic-spline extrapolation. Results are shown as solid symbols and dashed lines are guides for the eye only.

In regard to the specific heat for thorium mononitride, there are some older experimental data [56-58] but also some very recently published measurements [6,7]. In addition, semiempirical molecular-dynamics (MD) studies [9,10] and quasi-harmonic modeling [11,12] are reported. All these data are plotted in Figure 4 where we also show our calculated $C_{p}$ from lattice vibrations only $\left(C_{p}\right.$-lat $)$ and with the electronic contribution added $\left(C_{p}\right.$-lat-el). $\mathrm{ThN}$ is not magnetic so there is no magnetic contribution to $C_{p}$ for $\mathrm{ThN}$.

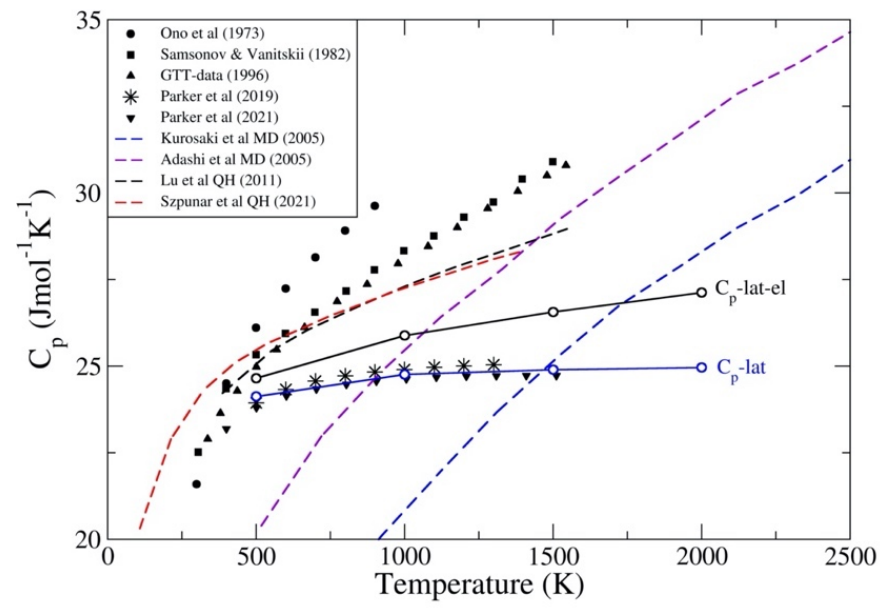

Figure 4. Specific heat at constant pressure, $C_{p}$, for ThN. Levels of theory are open circles, where the connecting lines are guides for the eye only. The dashed lines refer to semi-empirical moleculardynamics (MD) $[9,10]$ and quasi-harmonic $(\mathrm{QH})$ modeling [11,12]. Solid symbols are from experiments $[6,7,56-58]$.

Interestingly, our full theory $\left(C_{p}\right.$-lat-el $)$ is centered between the set of newer $[6,7]$ and older [56-58] experimental data. In this particular case, $\mathrm{QH}$ theory is not in reasonable agreement, not even below $1000 \mathrm{~K}$, with our anharmonic model. The semi-empirical MD results are also not in agreement with either our theory or any of the experimental data sets. We have no good explanations for the drastic variations we find between either the different experiments or the different modeling approaches for the specific heats displayed in Figure 4. We believe, however, that our results are sensible as they are positioned in the middle between experiments and other modeling. Certainly, more experimental 
and theoretical work may be required to better understand why these various data sets are so different.

\subsection{UC and UN}

For uranium monocarbide and mononitride there are considerably more experimental data available and consequently robust CALPHAD assessments can be done for these compounds [59-62]. This is very helpful for the theoretical modeling because we can directly compare the free energies for the two approaches. Otherwise, the free energy is a quantity that is not directly measured. For the thorium systems, on the other hand, we correlated our approach only with lesser quasi-harmonic theory that tends to lose quality at higher temperatures.

The only difference in our modeling of the uranium monocarbides and mononitrides, relative to their thorium counterparts, is the explicit inclusion of magnetic contributions to the free energy and the heat capacity. Admittedly, these contributions are rather small and in our previous study on UN [8] we did not consider them, but they do improve the agreement with CALPHAD as we shall see below. Otherwise, our theory for UN is the same as in our previous study [8].

First, we consider uranium monocarbide, UC. In Figure 5, we compare our present free energy with the results from the CALPHAD analysis. Apparently, the agreement is excellent between the two approaches, giving credence to both. For the theoretical model, the free energy has contributions from, in order of importance, lattice vibrations, electronic excitations, and magnetic entropy. Their individual relevance is reflected in the heat capacity, $C_{p}$ (see below). Indeed, the magnetic contributions are rather small, we show its contribution to the heat capacity in Figure 6, and it slightly improves the comparison with the CALPHAD free energy.

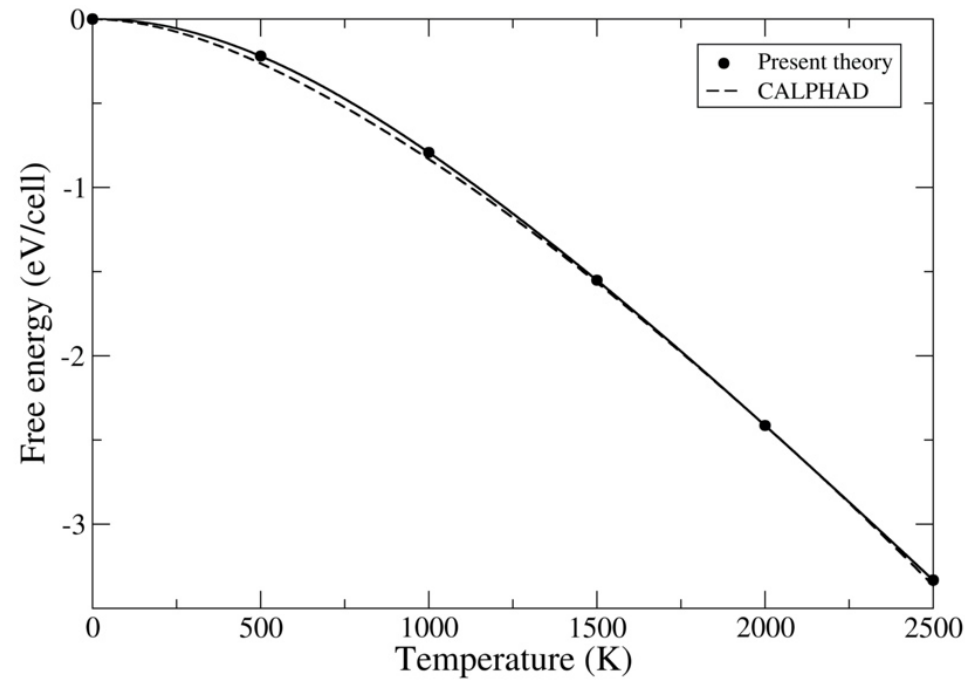

Figure 5. Free energy for UC from present theory together with results from our CALPHAD calculation (dashed line). Solid symbols show our present theory, and the solid line is guide for the eye only.

The specific heat has been measured numerous times for uranium monocarbide [63-66]. Here, we are primarily concerned with the high-temperature $C_{p}$ and focus our attention above 500 K. In Figure 6, we display experimental data [63-66], two CALPHAD assessments, and our present theory. The cause of the difference between the two CALPHAD results is the inclusion of defects, such as vacancies, on the carbon sublattice in one of the assessments. The presence of defects results in non-stoichiometry, which sensitively affects the heat capacity at elevated temperatures [59].

We see from our theoretical treatment in Figure 6 that the main contribution to $C_{p}$ is from lattice vibrations, but the electronic contribution is very important too. The full theory 
$\left(C_{p}\right.$-lat-el- $\left.m\right)$ also includes a small magnetic correction due to the magnetic-disorder entropy at high temperatures. It is rewarding to find in the figure that the CALPHAD approach, that assumes no defects (stoichiometric UC), is very close to our full theory. We do not expect the CALPHAD analysis that includes strong defect effects to agree with our theory because our model does not deal with any defect mechanisms but rather assumes a perfect, stoichiometric, crystal.

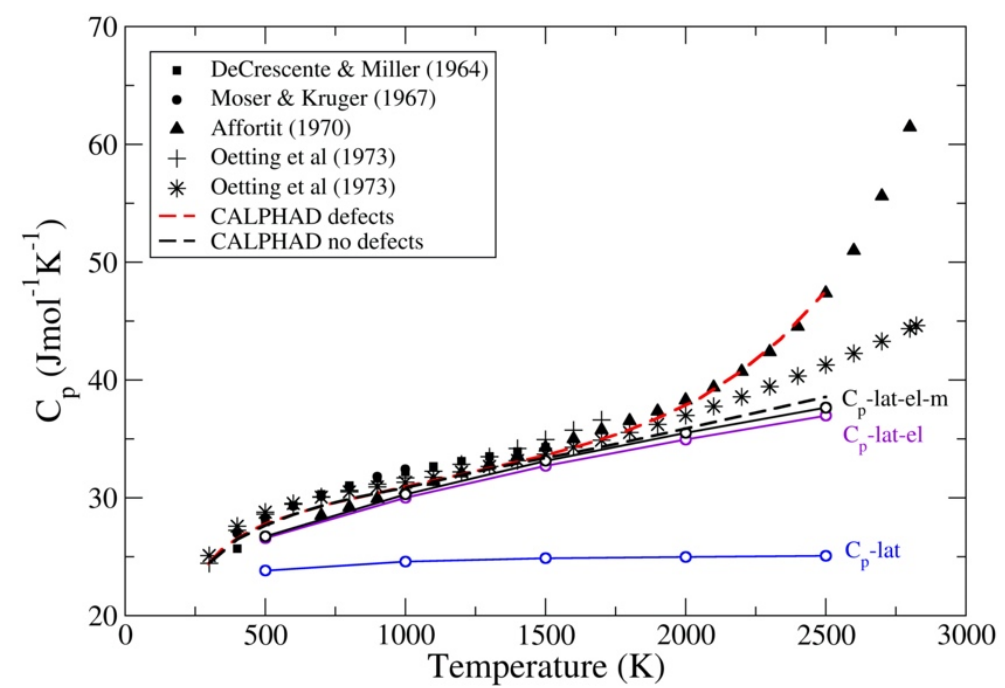

Figure 6. Specific heat at constant pressure, $C_{p}$, for UC. Levels of theory are open circles, where the connecting lines are guides for the eye only. Solid symbols are experimental data from [63-66] and CALPHAD results are dashed lines.

Moving on to uranium mononitride, we acknowledge that there is a fair amount of high-temperature data on this compound [7,67-73]. Before we reflect on the heat capacity, we once more compare the free energies from CALPHAD and our model, that includes a magnetic contribution. In Figure 7, we plot these energies and notice a very good consistency between them. This figure is very similar to a corresponding figure in [8], but in the present free energy for UN we also include the magnetic contribution. The magnetic entropy is increasingly important at higher temperatures, and its consideration significantly improves the agreement with CALPHAD in the high-temperature regime.

Hence, for the two uranium compounds, our first-principles anharmonic treatment is in excellent agreement with CALPHAD for the free energies.

Another test of the model is the comparison with the many sets of experimental heat capacities that have been reported for uranium mononitride [7,67-73]. In Figure 8, we show the results from those experimental works as well as our CALPHAD data and present theory.

This figure is rather busy with many data points and here we indicate our present theory as a solid line without showing the actual data points (1000, 1500, 2000, 2500, and $3000 \mathrm{~K})$.

The magnetic contribution to the heat capacity (not shown) for UN is calculated to be very small even though the net magnetic moment is significant, see Table 2 . This is so because the temperature dependence on the magnetic moment is calculated to be very small. Higher temperatures usually suppress magnetism due to the associated thermal broadening of the electronic density of states. Conversely, the thermal volume expansion tends to enhance magnetism and we find that in this case for UN, these two opposing effects neutralize each other, resulting in a very weak thermal dependence of the magnetism. 


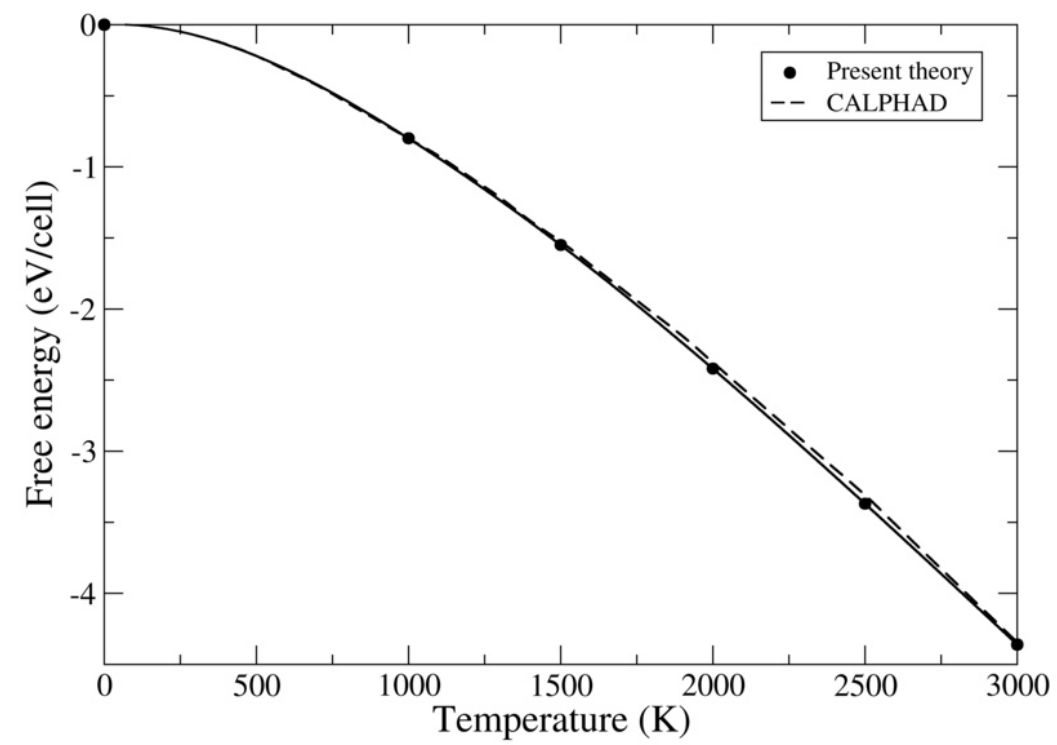

Figure 7. Free energy for UN from present theory together with results from our CALPHAD calculation (dashed line). Solid symbols show our present theory, and the solid line is guide for the eye only.

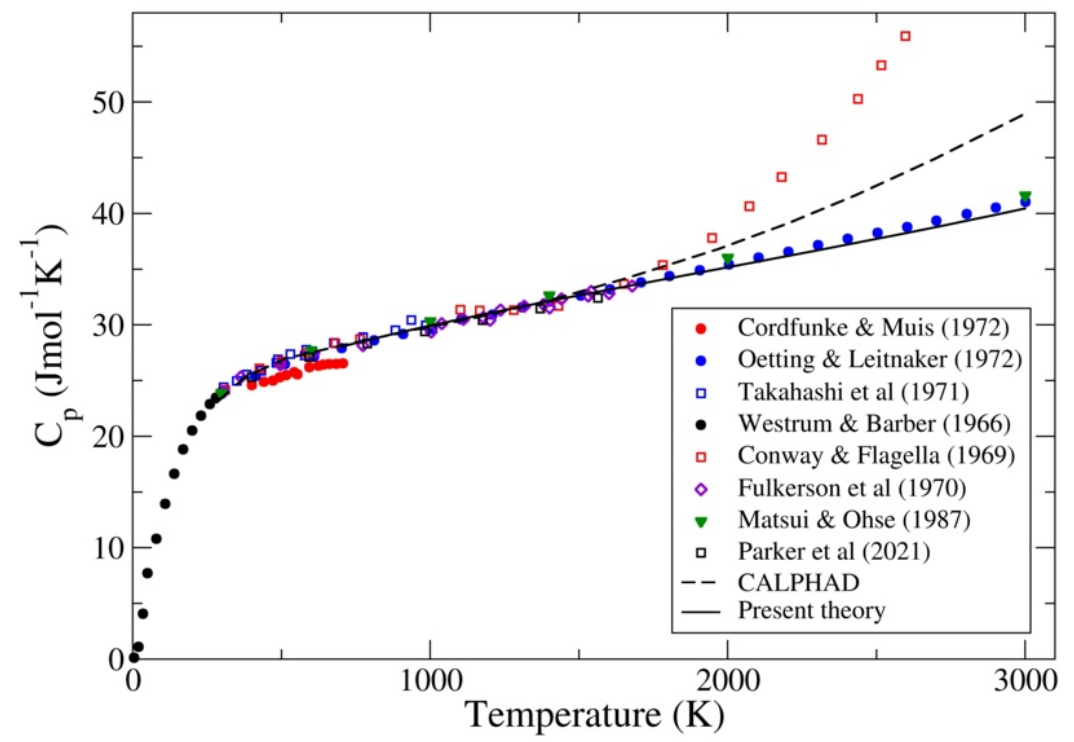

Figure 8. Specific heat at constant pressure, $C_{p}$, for UN. Present theory is here indicated by the solid line. Experimental data are from [7,67-73] and CALPHAD data are shown with a dashed line.

\section{3. $P u C$ and $P u N$}

We now focus on the plutonium monocarbide and mononitride. For these compounds we are again able to use CALPHAD assessments [59-62] of the available experimental data to help us better evaluate the validity and accuracy of our first-principles model. One difference we notice in the modeling between $\mathrm{PuC}$, and $\mathrm{PuN}$ is that, although both are magnetic, the effect of magnetism on the thermodynamics is significantly stronger for PuN. The explanation is that the spin and orbital magnetic moments in PuC are very nearly of the same magnitude, see Table 2, and anti-parallel. Hence, the net magnetic moment is small for PuC. Actually, this situation is reminiscent of the magnetic cancellation that has been proposed to exist in $\delta$-phase plutonium [74]. For PuN, on the other hand, our ferromagnetic calculations suggest a substantially larger spin than orbital moment so that the resulting net magnetic moment is considerable. 
We recently studied the thermodynamics for plutonium monocarbide [15] and the results from that investigation are included here with only minor modifications. We have here added the small magnetic contribution to the free energy. In Figure 9, we show the free energies from our full theory (lattice, electronic, and magnetic contributions) and the results from the CALPHAD computations. We notice that the agreement is not quite as good as it is for the uranium compounds shown above, especially at higher temperatures. This is also the case, as we shall see, for PuN. The reason for these discrepancies in the free energies, particularly at the highest temperatures, could be due to either the first-principles theory, the CALPHAD, or both. We will discuss some possible causes below.

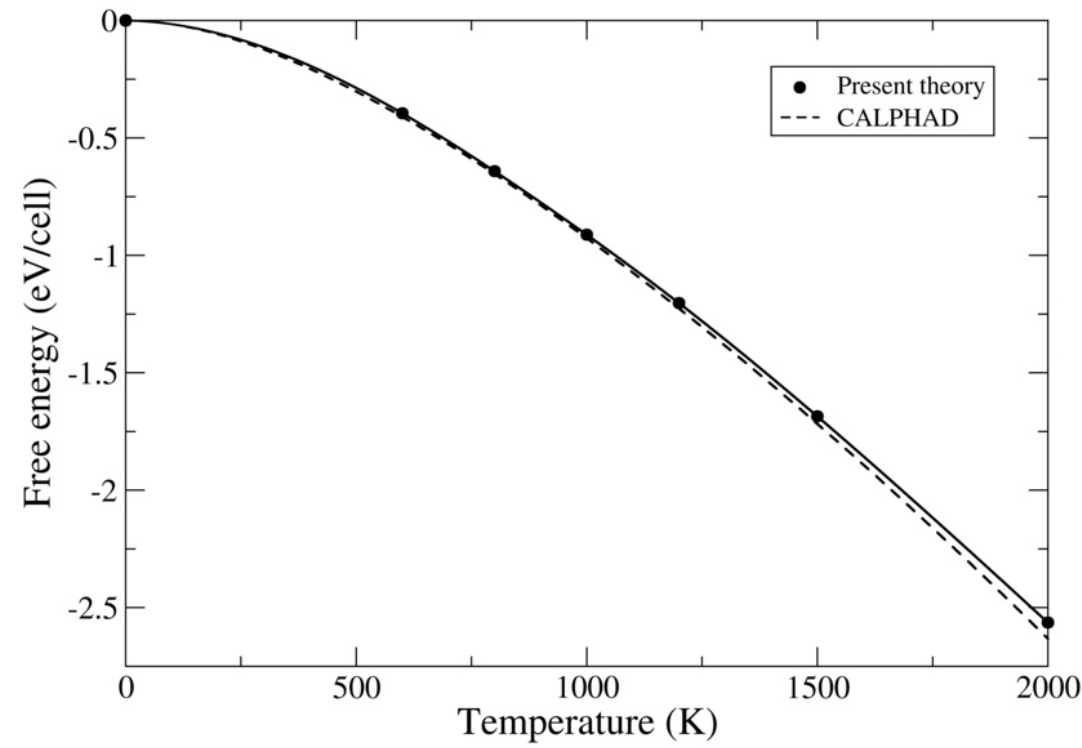

Figure 9. Free energy for PuC from present theory together with results from our CALPHAD calculation (dashed line). Solid symbols show our present theory, and the solid line is guide for the eye only.

As is the case for the uranium compounds, there are experimental data available for the thermodynamical properties of plutonium monocarbide [75-77]. In Figure 10 we show the heat capacities from these experimental studies and also the results from two CALPHAD assessments for different stoichiometries of $\mathrm{PuC}$. The calculated $C_{p}$ shown here is the same as published [15] because the magnetic contribution to $C_{p}$ is negligible. Our calculated $C_{p}$ is relatively consistent with the older measurement [75] and the CALPHAD data for the ideal-stoichiometric PuC. We believe carbon deficiency plays a major role for these experiments but acknowledge that the present theoretical framework does not take any such effects into account.

Next, we report our results for plutonium mononitride. As before, we scrutinize our theory by carefully comparing the free energy with the result from CALPHAD, in Figure 11. Analogous to $\mathrm{PuC}$, our first-principles free energy for $\mathrm{PuN}$ is slightly greater than the CALPHAD free energy at higher temperatures. We have already touched on the issue of stoichiometry as a possible cause of the discrepancy, but there could also be other sources for the differences that we shall discuss below. 


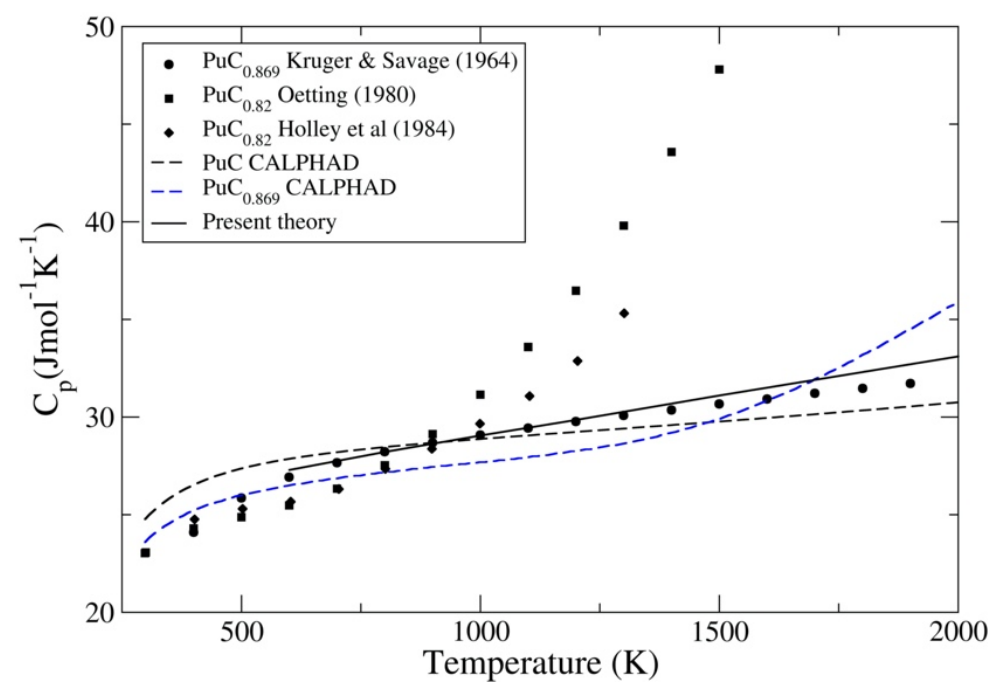

Figure 10. Specific heat at constant pressure, $C_{p}$, for PuC. Present theory is here indicated by the solid line. Experimental data are from [75-77] and CALPHAD data are shown with a dashed line, corresponding to stoichiometric $\mathrm{PuC}$ and sub-stoichiometric $\mathrm{PuC}_{0.869}$.

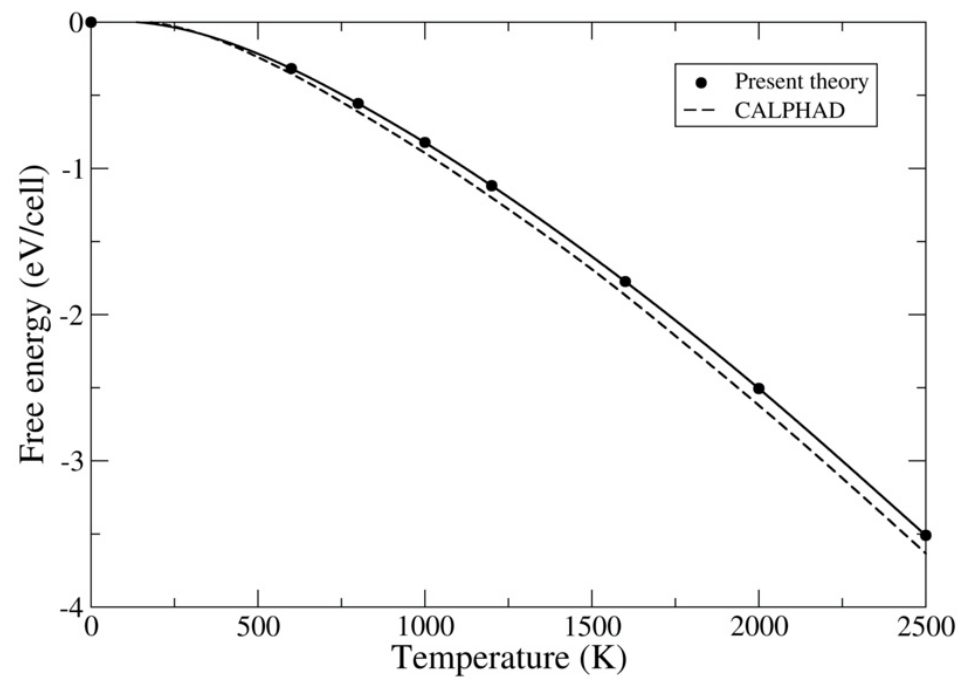

Figure 11. Free energy for PuN from present theory together with results from our CALPHAD calculation (dashed line). Solid symbols show our present theory, and the solid line is guide for the eye only.

We conclude by discussing our results on the specific heat of plutonium mononitride. There are some published reports of measured data $[13,78,79]$, in addition to a semiempirical-model's results [9], that we can compare with, apart from our CALPHAD data. In Figure 12, we show these heat capacities and our present theory. The three sets of experimental data $[13,78,79]$ are inconsistent with each other, but the Oetting results [78] agree with the MD simulations [9] and the CALPHAD data.

In regard to our theory, we show in Figure 12 the specific heat at constant volume obtained from the lattice-dynamics computations $\left(C_{v}\right.$-lat $)$ to highlight the rather strong volume dependence of the specific heat. For the other cases we have presented in this report, the difference between $C_{v}$ and $C_{p}$ is smaller (not shown). Notice that both the electronic $\left(C_{p}\right.$-lat-el) and the magnetic $\left(C_{p}\right.$-lat-el-m) contributions are significant. The full theory $\left(C_{p}\right.$-lat-el-m) is rather close to Oetting [79] and the MD data [9]. It is gratifying to also find very good agreement with our CALPHAD results and we emphasize that including the magnetic interaction is essential for good accuracy of $C_{p}$ for PuN. 


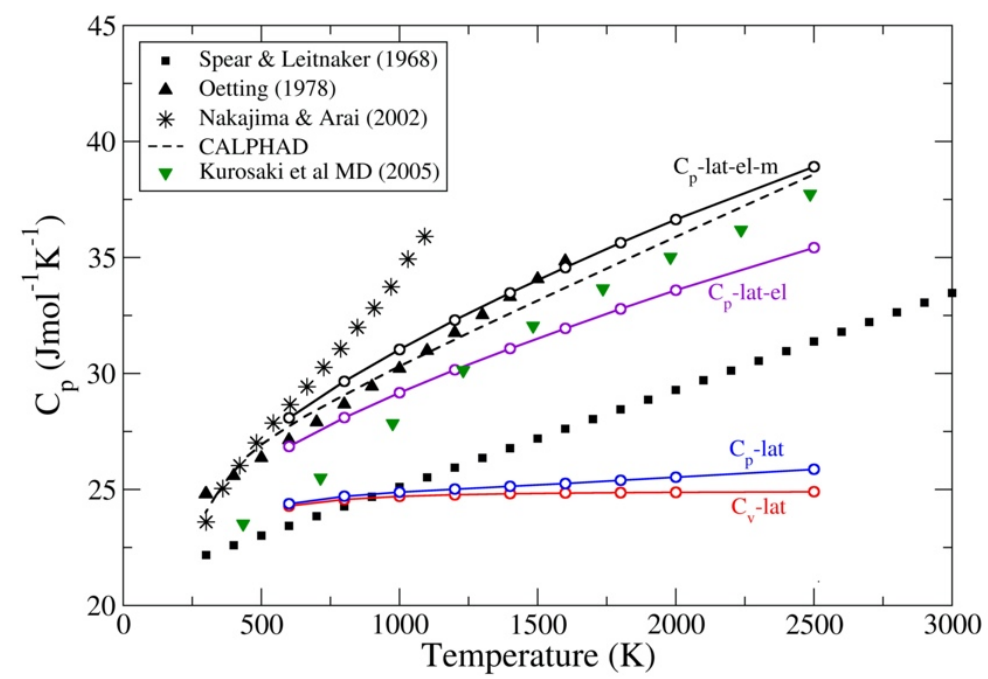

Figure 12. Specific heat at constant pressure, $C_{p}$, for PuN. The levels of theory are indicated by open circles. The connecting lines are guide for the eye only. Experimental data $[13,78,79]$ are shown as solid black symbols, the semi-empirical molecular-dynamics (MD) simulations [9] as green triangles, and CALPHAD data with a dashed line.

\section{Discussion and Conclusions}

We have studied the actinide monocarbides and mononitrides ThC, ThN, UC, UN, $\mathrm{PuC}$, and PuN, focusing on the thermodynamics at higher temperatures. The approach is that of a fully relativistic density-functional theory that is extended to include orbital polarization for the electronic structure. The lattice dynamics is obtained from temperaturedependent self-consistent phonons that couple in a strongly anharmonic fashion. Our modeling approach is more accurate than most others we have found for these materials. In the literature, first-principles theory of lattice dynamics of these actinide compounds has been restricted to be quasi-harmonic, except our own studies for UN [8] and PuC [15].

In addition to our first-principles thermodynamics model, we performed CALPHAD computations for the uranium and plutonium compounds for which more experimental data are available than for the thorium compounds. The CALPHAD results satisfactorily reproduced the experimental data such as the heat capacity.

Concentrating on the ambient-pressure, temperature dependence of the free energy and specific heat, we related our relativistic-anharmonic theory with CALPHAD and experiments, where available, or quasi-harmonic modeling. For ThC and ThN, our free energies were consistent with reported quasi-harmonic theory at temperatures up to about $1500 \mathrm{~K}$ but appeared to deviate above that. There are no high-temperature specific-heat data for ThC, and for $\mathrm{ThN}$ they are sparse and inconsistent at higher temperatures. For ThN, our predictions of $C_{p}$ are centered between several experimental data sets, suggesting they are reasonable. However, more experimental work is required for a better understanding of the disparity between the various measured data sets of the heat capacity for ThN.

In the case of the uranium compounds, UC and UN, more data are available, and we have available CALPHAD assessments for both. The first-principles free energies agree excellently with CALPHAD, and the calculated specific heats are consistent with some (not all) experimental data and CALPHAD. We found, however, that the inclusion of defects in the CALPHAD modeling sensitively impacts the specific heat, thus complicating the comparisons with our defect-free theory.

For PuC and PuN, the free-energy discrepancy between theory and CALPHAD is greater than for their uranium counterparts. The difference is relatively small (less than $3 \%$ ) but non-negligible. The reason could be inaccuracies in either the first-principles modeling or the CALPHAD approach, where the latter, semi-empirical models are reliant on the availability of experimental data. In terms of theory, it is plausible that electronphonon coupling, that is not fully explored here, is underestimated for the plutonium 
compounds. That would then explain why the theoretical free energy is slightly above that of CALPHAD. Another issue is the question of stoichiometry of the compound, which can be accounted for using the CALPHAD method with vacancies on the carbon or the nitrogen sublattice. We make no attempts to account for possible non-stoichiometric states in the theory, however. Lastly, it is conceivable that fundamental electron-correlation effects in the plutonium compounds are not sufficiently addressed in the present electronic structure model. However, for PuC we discussed this possibility at some length [15] and showed that the DFT + OP approach for the electronic structure is largely appropriate, and we believe this conclusion holds true also for PuN.

Finally, measured phonon properties for these actinide compounds are very scarce and therefore it is useful for other modeling and for reference to show our calculated phonon density of states (DOS). We chose not to illustrate all the phonon dispersions for the compounds as that would require many more figures, but they indicate positive phonon frequencies everywhere, ensuring dynamical stability for all compounds. Instead, we show, in Figure 13, phonon DOS at $2000 \mathrm{~K}$ for ThC, ThN, UC, UN, PuC, and PuN. In this figure we only display the states associated with the acoustic frequencies. The phonon DOS for all compounds look quantitatively similar with a double-peak feature and they are also not very different from the phonon DOS of $\delta$-phase plutonium.

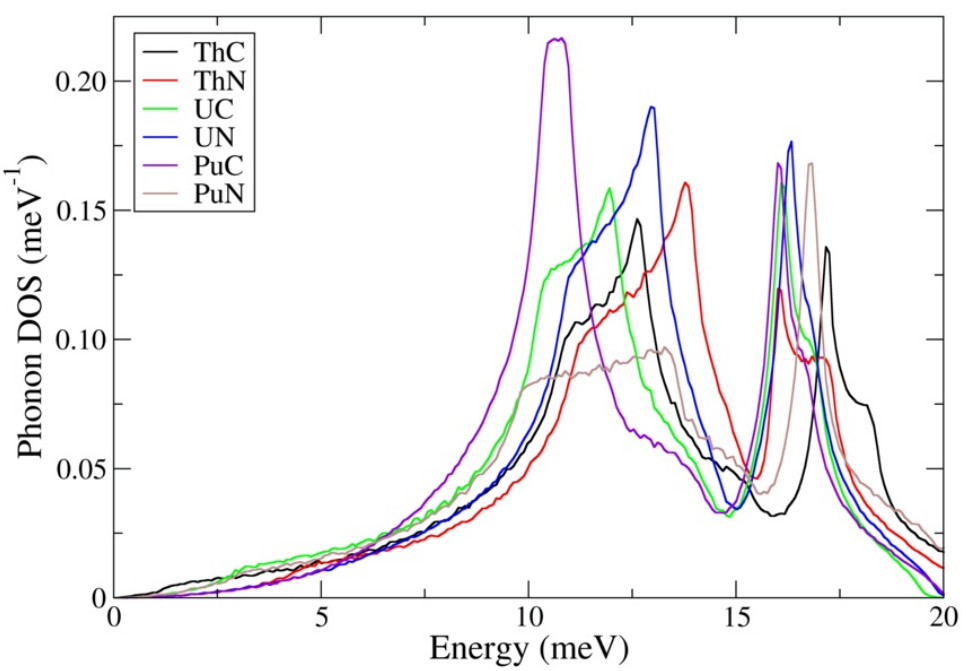

Figure 13. SCAILD phonon density of states (states/meV) at $2000 \mathrm{~K}$ for ThC, ThN, UC, UN, PuC, and PuN.

Author Contributions: Conceptualization, P.S.; methodology, P.S., E.E.M.; software, P.S., E.E.M.; investigation, P.S., E.E.M. writing-original draft preparation, P.S.; writing-review and editing, P.S., E.E.M., C.J.W. All authors have read and agreed to the published version of the manuscript.

Funding: This research received no external funding.

Institutional Review Board Statement: Not applicable.

Informed Consent Statement: Not applicable.

Data Availability Statement: Not applicable.

Acknowledgments: We acknowledge helpful discussions with B. Sadigh and A. Landa. This work was performed under the auspices of the U.S. DOE by LLNL under Contract DE-AC52-07NA27344.

Conflicts of Interest: The authors declare no conflict of interest.

\section{References}

1. Abram, T.; Ion, S. Generation-IV nuclear power: A review of the state of the science. Eng. Policy 2008, 36, 4323-4330. [CrossRef]

2. Matzke, H. Science of Advanced LMFBR Fuels; North-Holland: Amsterdam, The Netherlands, 1986. 
3. Mishra, V.; Chaturvedi, S. Thermophysical properties of thorium compounds from first principles. In Proceedings of the Thorium energy conference 2015-ThEC15, Mumbai, India, 12-15 October 2015. Available online: http:/ / www.thoriumenergyworld.com/ uploads/6/9/8/7/69878937/thermophysical_properties_of_thorium_compounds_from_first_principles_thec15_paper.pdf (accessed on 1 December 2021).

4. Sahafi, M.H.; Mahdavi, M. Ab-initio investigations on dynamical and lattice thermal behaviours of ThC. Bull. Mater. Sci. 2021, 44, 1-13. [CrossRef]

5. Kývala, L.; Legut, D. Lattice dynamics and thermal properties of thorium metal and thorium monocarbide. Phys. Rev. B 2020, 101, 1-14. [CrossRef]

6. $\quad$ Parker, S.S.; White, J.T.; Hosemann, P.; Nelson, A.T. Thermophysical properties of thorium mononitride from 298 to 1700 K. J. Nucl. Mater. 2019, 526, 1-9. [CrossRef]

7. $\quad$ Parker, S.S.; Newman, S.; Fallgren, A.J.; White, J.T. Thermophysical properties of mixes of thorium and uranium nitride. JOM 2021, 73, 3564-3575. [CrossRef]

8. Söderlind, P.; Landa, A.; Perron, A.; Sadigh, B.; Heo, T.W. Ground-state and thermodynamical properties of uranium mononitride from anharmonic first-principles theory. Appl. Sci. 2019, 9, 3914. [CrossRef]

9. Kurosaki, K.; Adachi, J.; Uno, M.; Yamanaka, S. Molecular dynamics studies of actinide nitrides. J. Nucl. Mater. 2005, 344, 45-49. [CrossRef]

10. Adashi, J.; Kurosaki, K.; Uno, M.; Yamanaka, S. A molecular dynamics study of thorium nitride. J. Alloys Compd. 2005, 394, 312-316. [CrossRef]

11. Lu, Y.; Li, D.-F.; Wang, B.-T.; Li, R.-W.; Zhang, P. Electronic structures, mechanical and thermodynamical properties of ThN from first-principles calculations. J. Nucl. Mater 2011, 408, 136-141. [CrossRef]

12. Szpunar, B.; Ranasinghe, J.I.; Malakkal, L.; Szpunar, J.A. First principles investigation of thermal properties of thorium mononitride. J. Alloys Compd. 2021, 879, 160467-1-160467-8. [CrossRef]

13. Nakajima, K.; Arai, Y. Heat capacity of neptunium mononitride. J. Nucl. Sci. Technol. 2002, 39, 620-623. [CrossRef]

14. Péres Daroca, D.; Jaroszewicz, S.; Llois, A.M.; Mosca, H.O. First-principles study of point defects in thorium carbide. J. Nucl. Mater. 2014, 454, 217-222. [CrossRef]

15. Söderlind, P.; Landa, A.; Perron, A.; Moore, E.E.; Wu, C. Thermodynamics of plutonium monocarbide from anharmonic and relativistic theory. Appl. Sci. 2020, 10, 6524. [CrossRef]

16. Lai, C.; Hu, Y.; Qiu, R. Thermodynamical stability of substoichiometric plutonium monocarbide from first-principles calculations. Phys. Chem. Chem. Phys. 2020, 22, 9009-9013. [CrossRef]

17. Lai, C.; Hu, Y.; Qiu, R. Exploring the sub-stoichiometric behavior of plutonium mononitride. RSC Adv. 2020, 10, $24877-24881$. [CrossRef]

18. Kocevski, V.; Rehn, D.A.; Cooper, M.W.D.; Anderson, D.A. First-principles investigation of uranium mononitride (UN): Effect of magnetic ordering, spin-orbit interactions and exchange correlation functional. J. Nucl. Mater. 2021, 559, 153401. [CrossRef]

19. Johansson, B.; Ahuja, R.; Eriksson, O.; Wills, J.M. Anomalous fcc crystal structure of thorium metal. Phys. Rev. Lett. 1995, 75, 280-283. [CrossRef]

20. Söderlind, P. First-principles phase stability, bonding, and electronic structure of actinide metals. J. Electron Spectr. Rel. Phenom. 2014, 194, 2-7. [CrossRef]

21. Wen, X.-D.; Martin, R.L.; Scuseria, G.E.; Rudin, S.P.; Batista, R.E. A screened hybrid DFT study of actinide oxides, nitrides, and carbides. J. Phys. Chem. C 2013, 117, 13122-13128. [CrossRef]

22. Li, R.-S.; Tong, N.-H.; Wang, J.-T.; Xin, D.-Q.; Huang, S.-Q. A first principle calculation on electronic properties of plutonium mononitride: Insights from dynamical mean field theory. J. Nucl. Mater. 2018, 511, 277-283. [CrossRef]

23. Wdowik, U.D.; Piekarz, P.; Legut, D.; Jaglo, G. Effect of spin-orbit and onsite Coulomb interactions on the electronic structure and lattice dynamics of uranium monocarbide. Phys. Rev. B 2016, 94, 054303-1-054303-8. [CrossRef]

24. Eriksson, O.; Brooks, M.S.S.; Johansson, B. Orbital polarization in narrow-band systems: Application to volume collapses in light lanthanides. Phys. Rev. B 1990, 41, 7311-7314. [CrossRef] [PubMed]

25. Eschrig, H.; Sargolzaei, M.; Koepernik, K.; Richter, M. Orbital polarization in Kohn-Sham-Dirac theory. EPL 2005, 72, 611-617. [CrossRef]

26. Solovyev, I.V.; Liechtenstein, A.I.; Terakura, K. Is Hund's second rule responsible for the orbital magnetism in solids? Phys. Rev. Lett. 1998, 80, 5758-5761. [CrossRef]

27. Söderlind, P.; Landa, A.; Sadigh, B. Density-functional theory for plutonium. Adv. Phys. 2019, 68, 1-47. [CrossRef]

28. Sadigh, B.; Kutepov, A.; Landa, A.; Söderlind, P. Assessing relativistic effects and electron correlation in the actinide metals Th-Pu. Appl. Sci. 2019, 9, 5020. [CrossRef]

29. Shein, I.R.; Ivanovskii, A.L. The influence of carbon non-stoichiometry on the electronic properties of thorium monocarbide ThC. Solid State Sci. 2010, 12, 1580-1584. [CrossRef]

30. Wills, J.M.; Eriksson, O.; Andersson, P.; Delin, A.; Grechnyev, O.; Alouani, M. Full-Potential Electronic Structure Method; Springer Science and Business Media LLC: Berlin, Germany, 2010.

31. Gerward, L.; Staun Olsen, J.; Benedict, U.; Itíe, J.-P.; Spirlet, J.C. Structural stability and equation of state of thorium carbide for pressures up to 36 GPa. J. Appl. Cryst. 1986, 19, 308-310. [CrossRef] 
32. Gerward, L.; Staun Olsen, J.; Benedict, U.; Itíe, J.-P.; Spirlet, J.C. The crystal structure and equation of state of thorium nitride for pressures up to $47 \mathrm{GPa}$. J. Appl. Cryst. 1985, 18, 339-341. [CrossRef]

33. Vigier, N.; Auwer, D.; Fillaux, C.; Maslennikov, A.; Noël, H.; Roques, J.; Shuh, D.K.; Simoni, E.; Tyliszczak, T.; Moisy, P. New data on the structure of uranium monocarbide. Chem. Mater. 2008, 20, 3199-3204. [CrossRef]

34. Staun Olsen, J.; Gerward, L.; Benedict, U. A new high-pressure phase of uranium nitride studied by X-ray diffraction and synchrotron radiation. J. Appl. Cryst. 1985, 18, 37-41. [CrossRef]

35. Boeuf, A.; Caciuffo, R.; Fournier, J.M.; Manes, L.; Rebizant, J.; Roudaut, E.; Rustichelli, F. ${ }^{239}$ PuN powder neutron diffraction study. Solid State Comm. 1984, 52, 451-453. [CrossRef]

36. Liang, Y.; Zhang, W.; Chen, L. Phase stability and mechanical properties of two new carbon crystals. EPL 2009, 87, 56003-1-56003-6. [CrossRef]

37. Green, J.L.; Arnold, G.P.; Leary, J.A.; Nereson, N.G. Crystallographic and magnetic ordering studies of plutonium carbides using neutron diffraction. J. Nucl. Mater. 1970, 34, 281-289. [CrossRef]

38. Curry, N.A. An investigation of the magnetic structure of uranium nitride by neutron diffraction. Proc. Phys. Soc. 1965, 86, 1193-1198. [CrossRef]

39. Söderlind, P. Quantifying the importance of orbital over spin correlations in $\delta$-Pu within density-functional theory. Phys. Rev. B 2008, 77, 1-5. [CrossRef]

40. Blöchl, P.E. Projector augmented-wave method. Phys. Rev. B 1994, 50, 17953-17979. [CrossRef]

41. Kresse, G.; Furthmüller, J. Efficient iterative schemes for ab initio total-energy calculations using a plane-wave basis set. Phys. Rev. B 1996, 54, 11169-11186. [CrossRef] [PubMed]

42. Kresse, G.; Joubert, D. From ultrasoft pseudopotentials to the projector augmented-wave method. Phys. Rev. B 1999, 59, 1758-1775. [CrossRef]

43. Souvatzis, P.; Eriksson, O.; Katsnelson, M.; Rudin, S. The self-consistent ab initio lattice dynamical method. Comput. Mater. Sci. 2009, 44, 888-894. [CrossRef]

44. Kaufman, L.; Bernstein, H. Computer Calculation of Phase Diagrams with Special Reference to Refractory Metals; Academic Press: New York, NY, USA, 1970.

45. Saunders, N.; Miodownik, A. CALPHAD Calculation of Phase Diagrams: A Comprehensive Guide; Elsevier Science: Amsterdam, The Netherlands, 1998.

46. Lukas, H.; Fries, S.; Sundman, B. Computational Thermodynamics: The CALPHAD Method; Cambridge University Press: Cambridge, UK, 2007.

47. Ravat, B.; Oudot, B.; Perron, A.; Lalire, F.; Delaunay, F. Phase transformations in PuGa 1at.\% alloy: Study of whole reversion process following martensitic transformation. J. Alloys Compd. 2013, 580, 298-309. [CrossRef]

48. Perron, A.; Ravat, B.; Oudot, B.; Lalire, F.; Mouturat, K.; Delaunay, F. Phase transformations in Pu-Ga alloy: Synergy between simulations and experiments to elucidate direct and indirect reversion competition. Acta Mater. 2013, 61, 7109-7120. [CrossRef]

49. Perron, A.; Turchi, P.; Landa, A.; Söderlind, P.; Ravat, B.; Oudot, B.; Delaunay, F.; Kurata, M. Thermodynamic re-assessment of the $\mathrm{Pu}-\mathrm{U}$ system and its application to the ternary Pu-U-Ga system. J. Nucl. Mater. 2014, 454, 81-95. [CrossRef]

50. Perron, A.; Turchi, P.; Landa, A.; Söderlind, P.; Ravat, B.; Oudot, B.; Delaunay, F. The Pu-U-Am system: An ab initio informed CALPHAD thermodynamic study. J. Nucl. Mater. 2015, 458, 425-441. [CrossRef]

51. Moore, E.E.; Turchi, P.E.A.; Landa, A.; Söderlind, P.; Oudot, B.; Belof, J.L.; Stout, S.A.; Perron, A. Development of a CALPHAD thermodynamic database for Pu-U-Fe-Ga alloys. Appl. Sci. 2019, 9, 5040. [CrossRef]

52. Grimvall, G. Spin disorder in paramagnetic fcc iron. Phys. Rev. B 1989, 39, 12300-12301. [CrossRef] [PubMed]

53. Wang, Y. Classical mean-field approach for thermodynamics: Ab initio thermophysical properties of cerium. Phys. Rev. B 2000, 61, R11863-R11866. [CrossRef]

54. Danan, J. Chaleur specifique de 2 a $300 \mathrm{~K}$ monocarbure de thorium. J. Nucl. Mater. 1975, 57, 280-282. [CrossRef]

55. Grimvall, G.; Häglund, J.; Fernández Guillermet, A. Spin fluctuations in paramagnetic chromium determined from entropy considerations. Phys. Rev. B 1993, 47, 15338-15341. [CrossRef]

56. Ono, F.; Kanno, M.; Mukaibo, T. Heat capacity of thorium nitrides from 450 to 800K. J. Nucl. Sci. Technol. 1973, 10, 391-395. [CrossRef]

57. Samsonov, G.V.; Vanitskii, I.M. Handbook of Refractory Metals; Plenum Publishing Corporation: New York, NY, USA, 1982.

58. The SGTE Pure Substance and Dolution Database, GTT-Data Services. 1996. Available online: http://www.thermocalc.com/ TCDATA.htm (accessed on 1 December 2021).

59. Guéneau, C.; Dupin, S.; Sundman, B.; Martial, C.; Dumas, J.-C.; Gossé, S.; Chatain, S.; De Bruycker, F.; Manara, D.; Konings, R.J.M. Thermodynamic modelling of advanced oxide and carbide nuclear fuels: Description of the U-Pu-O-C systems. J. Nucl. Mater. 2011, 419, 145-167. [CrossRef]

60. Chevalier, P.-Y.; Fischer, E.; Cheynet, B. Thermodynamic modelling of the N-U system. J. Nucl. Mater. 2000, 280, 136-150. [CrossRef]

61. Besmann, T.; Shin, D.; Lindemer, T.B. Uranium nitride as LWR TRISO fuel: Thermodynamic modeling of U-C-N. J. Nucl. Mater. 2012, 427, 162-168. [CrossRef]

62. OECD NEA/NSC: Thermodynamics of Advanced Fuels-International Database (TAF-ID), (n.d.). Available online: https: //www.oecd-nea.org/science/taf-id/taf-id-public/ (accessed on 7 November 2019). 
63. DeCresente, M.A.; Miller, A.D. High temperature properties of uranium carbide. In Carbides in Nuclear Energy; Russel, L.R., Ed.; Macmillan: London, UK, 1964; Volume 1, pp. 342-357.

64. Moser, J.B.; Kruger, O.L. Thermal conductivity and heat capacity of the monocarbide, monophosphide, and monosulfide of uranium. J. Appl. Phys. 1967, 38, 3215-3222. [CrossRef]

65. Affortit, C. Chaleur specifique de UC et UN. J. Nucl. Mater. 1970, 34, 105-107. [CrossRef]

66. Oetting, F.L.; Navratil, J.D.; Storms, E.K. The chemical thermodynamic properties of nuclear materials: (II) High temperature enthalpy of the uranium carbides. J. Nucl. Mater. 1973, 45, 271-283. [CrossRef]

67. Westrum, E.G.; Barber, C.M. Uranium mononitride: Heat capacity and thermodynamic properties from 5 to 350 K. J. Chem. Phys. 1966, 45, 635-638. [CrossRef]

68. Conway, J.B.; Flagella, P.N. Physical and Mechanical Properties of Reactor Materials; GEMP-1012; General Electric Company: Cincinnati, OH, USA, 1969.

69. Fulkerson, W.; Kollie, T.G.; Weawer, S.C.; Moore, J.P.; Williams, R.K. Plutonium 1970 and other Actinides Part I and II. In Proceedings of the 4th International Conference on Plutonium and other Actinides (AIME), Santa Fe, NM, USA, 5-9 October 1970.

70. Takahasi, Y.; Murabayashi, M.; Akimoto, Y.; Mukaibo, T. Uranium mononitride: Heat capacity and thermal conductivity from 298 to 1000 K. J. Nucl. Mater. 1971, 38, 303-308. [CrossRef]

71. Oetting, F.L.; Leitnaker, J.M. The chemical thermodynamic properties of nuclear materials I. Uranium mononitride. J. Chem. Thermodyn. 1972, 4, 199-211. [CrossRef]

72. Cordfunke, E.H.P.; Muis, R.P. The heat capacity of uranium mononitride. J. Nucl. Mater. 1972, 42, 233-234. [CrossRef]

73. Matsui, T.; Ohse, R.W. Thermodynamic properties of uranium nitride, plutonium nitride and uranium-plutonium mixed nitride. High Temp. High Press. 1988, 20, 169-175.

74. Söderlind, P.; Zhou, F.; Landa, A.; Klepeis, J.E. Phonon and magnetic structure in $\delta$-plutonium from density-functional theory. Sci. Rep. 2015, 5, 15958-1-15958-6. [CrossRef] [PubMed]

75. Kruger, O.L.; Savage, H. Heat capacity of plutonium monocarbide from $400^{\circ}$ to $1300^{\circ}$ K. J. Chem. Phys. 1964, 40, 3324-3328. [CrossRef]

76. Oetting, F.L. The chemical thermodynamics of nuclear materials. J. Nucl. Mater. 1980, 88, 265-272. [CrossRef]

77. Holley, C.E.; Rand, M.H.; Storms, E.K. The Chemical Thermodynamics of Actinide Elements and Compounds. Part 6: The Actinide Carbides; IAEA: Vienna, Austria, 1984; pp. 53-72.

78. Spear, K.E.; Leitnaker, J.M. A consistent set of thermodynamic values for plutonium mononitride. J. Am. Ceram. Soc. 1968, 51, 706-709. [CrossRef]

79. Oetting, F.L. The chemical thermodynamic properties of nuclear materials III: Plutonium mononitride. J. Chem. Thermodyn. 1978, 10, 941-948. [CrossRef] 